\title{
Role of IVUS in the rectification of angiographically judged ramus intermedius and its clinical significance
}

Xue Gong ${ }^{1,2+}$, Zheyong Huang ${ }^{2 \dagger}$, Zhonghan Sun ${ }^{3}$, Qibing Wang ${ }^{2}$, Juying Qian ${ }^{2}$, Lei Ge ${ }^{2^{*}}$ and Junbo Ge 2* $^{*}$

\begin{abstract}
Background: Due to the technical limitations of coronary artery angiography (CAG), ramus intermedius (RI) is sometimes difficult to distinguish from a high-origin obtuse marginal branch or a high-origin diagonal branch. This study sought to investigate the role of intravascular ultrasonography (IVUS) in the rectification of angiographically judged RI.

Methods: This study retrospectively analyzed 165 patients who were reported to have an RI based on CAG and underwent IVUS implementation from 02/01/2009 to 31/12/2019 in Zhongshan Hospital, Fudan University. Taking IVUS as the gold standard, we calculated the accuracy of RI identification by CAG and evaluated the impact of RI on revascularization strategy.

Results: Among the 165 patients, 89 patients (54\%) were demonstrated to have an RI on IVUS (IVUS-RI), 32 patients (19\%) were identified to have a high-origin diagonal branch on IVUS (IVUS-h-D), and 44 patients (27\%) had an actual high-origin obtuse marginal artery on IVUS (IVUS-h-OM). Among 84 patients who underwent one-stent crossover stenting because of left main furcation lesions (48 patients in the IVUS-RI group, 12 patients in the IVUS-h-D group, and 24 in the IVUS-h-OM group), 14.6\% of patients in the IVUS-RI group, 33.3\% in the IVUS-h-D group and 0\% in the IVUS-h-OM group had CAG-RI compromise $(P=0.02)$, which was defined as severe stenosis of the RI ostium (>75\%) or significant $\mathrm{Rl}$ flow impairment $(\mathrm{TIMl}<3)$.
\end{abstract}

Conclusions: Only 54\% of CAG-RIs were confirmed by IVUS, which indicates the necessity of preintervention IVUS to distinguish real RIs from other branches in LM furcation lesions.

Keywords: Ramus intermedius, Intravascular ultrasonography, Angiography, Coronary, Percutaneous coronary intervention

\section{Background}

The ramus intermedius (RI) is a variant coronary artery resulting from bifurcation of the left main coronary artery (LMCA) [1]. Generally, it is diagnosed on autopsy [2]. Recently, RI has increasingly been observed by

\footnotetext{
*Correspondence: ge.lei@zs-hospital.sh.cn; junboge@126.com

${ }^{\dagger}$ Xue Gong and Zheyong Huang: Co first authors.

2 Department of Cardiology, Shanghai Institute of Cardiovascular

Disease, Zhongshan Hospital, Fudan University, 180 Fenglin Road,

Shanghai 200032, People's Republic of China

Full list of author information is available at the end of the article
}

computed tomography angiography (CTA) of the coronary artery [3-5]. The occurrence rate is $\sim 20 \%$ (range $15-31 \%)$ of the population depending upon the series [3, 4].

Anatomically, RI is different from a high-origin obtuse marginal artery (h-OM) or a high-origin diagonal branch (h-D). Functionally, it is as important as these prominent early branches because it has a similar course and perfusion region to h-OM or h-D [1]. Once RI is occluded, patients can also have symptoms of chest pain, increased 
troponin levels and related electrocardiographic changes [6]. However, little attention has been paid to RI branch.

Percutaneous coronary intervention (PCI) with stent implantation has become a viable alternative to coronary artery bypass grafting (CABG) in patients with significant LM or LM bifurcation lesions [7]. However, PCI procedures for LM bifurcation lesions remain technically challenging [8-10]. The existence of RI changes an LM bifurcation lesion into a trifurcation lesion and changes the furcation angle [11], which makes PCI procedures more complicated. Therefore, it is important to distinguish RI from other prominent early branches.

Standard angiographic projections of coronary artery angiography (CAG) are often associated with vessel foreshortening and anatomical overlap [12]. This technique has a limited ability to capture the exact anatomy of the carina of the furcation or the ostial side branch (SB) [13]. Hence, it is reasonable to presume that many RIs reported on the basis of CAG do not truly originate from the furcation point. In contrast, intravascular ultrasonography (IVUS) is an accurate tomographic technique that is not affected by viewing angles; thus, it may overcome these shortcomings and might provide more valuable anatomical information than CAG [14].

The purpose of this study was to illustrate (1) the accuracy of CAG-reported RI using IVUS as the gold standard and (2) the impact of RI on revascularization strategy for the LM furcation.

\section{Methods}

\section{Study population}

Between January 2009 and December 2019, consecutive patients who had CAG-reported RI and underwent IVUS at Zhongshan Hospital, Fudan University, were enrolled. Patients were excluded in the event of suboptimal IVUS image quality or right coronary artery withdrawal. The study protocol was reviewed and approved by the Ethics Committee of Zhongshan Hospital, Fudan University. All patients provided written informed consent for the use of their data.

\section{Procedures and data collection}

All CAG procedures were performed using standard coronary angiography projections. The standard fluoroscopic views included right anterior oblique (RAO) $30^{\circ}$, RAO $30^{\circ}$ /cranial (CRA) $30^{\circ}$, RAO $30^{\circ}$ caudal (CAU) $30^{\circ}$, left anterior oblique (LAO) $45^{\circ} / \mathrm{CAU} 30^{\circ}$ ("spider" view), LAO $30^{\circ} / \mathrm{CRA} 30^{\circ}$ and CRA $30^{\circ}$ for the left coronary artery, as well as LAO $45^{\circ}$, LAO $20^{\circ} / \mathrm{CRA} 20^{\circ}$, and $\mathrm{RAO} 30^{\circ}$ for the right coronary artery. The "spider" view is often used to analyze the LMCA furcation and ostium of the left anterior descending artery (LAD) and left circumflex artery (LCX). If a SB can be seen coming out of the carina of LMCA furcation in the "spider" view, it will be reported as a RI by the cardiologists. PCI was performed according to the 2018 ESC/EACTS Guidelines on myocardial revascularization [15]. RI compromise was defined as severe stenosis of the RI ostium ( $>75 \%$ ) or significant RI flow impairment (TIMI <3) $[9,16]$. Each interventional cardiologist was responsible for the decision to employ a single or double stenting strategy for the treatment of LM furcation lesions. Demographic characteristics and clinical data were obtained from electronic medical record review.

\section{IVUS imaging acquisition}

IVUS was performed after a $200 \mu \mathrm{g}$ dose of intracoronary nitroglycerin using a commercially available imaging system (iMap, Boston Scientific, Natick, MA, USA), an automated motorized pullback system $(0.5 \mathrm{~mm} / \mathrm{s})$, and the corresponding $40 \mathrm{MHz}$ IVUS catheter (Atlantis SR Pro., Boston Scientific, Natick, MA, USA). After guidewire crossing, the IVUS catheter was carefully advanced $10 \mathrm{~mm}$ distal to the culprit lesion and was pulled back automatically to the LMCA ostium. Images were recorded continuously for offline analysis.

\section{IVUS analysis method}

Commercially available software (ImageViewer_05_14_2018_1, Boston Scientific, Corporation/Scimed, Natick, MA) was used. IVUS images of the distal LMCA and its branches were reviewed offline by an experienced observer who was blinded to individual patient data. The standards for the determination of IVUS-RI, IVUS-h-D and IVUS-h-OM on IVUS when withdrawing from LAD were as follows: if a SB could be seen at the entrance of LCX, and the three lumens had blood flow, the presence of RI was confirmed; if a SB was fully incorporated into the LAD before LCX entered, it was considered a h-D; if no SB could be seen within $2 \mathrm{~mm}$ before LCX entered, a h-OM was considered to be present.

\section{Statistical analysis}

All statistical analyses were conducted using $\mathrm{R}$ (version 3.5.1, https://www.r-project.org/). Continuous variables are shown as the mean \pm SD or median (IQR) according to the distribution of the data, and categorical variables are shown as $\mathrm{N}$ (\%). The $P$ values for intergroup differences were calculated using the Kruskal-Wallis test for continuous variables and the chi-squared test or Fisher's exact test for categorical variables. A $P$ value of less than 0.05 was considered to indicate statistical significance. 


\section{Results}

Patient enrollment and characteristics

Of the 107,505 patients referred for CAG from $02 / 01 / 2009$ to $31 / 12 / 2019,2679$ patients (2.5\%) were reported to have an RI, and 188 of those 2679 patients underwent IVUS. After the exclusion of patients with right coronary artery withdrawal (19 patients) or suboptimal IVUS image quality (4 patients), the study ultimately included 165 patients (the mean age was $64.8 \pm 10.4$ years old, and $16 \%$ were female). According to the results of their IVUS review, they were divided into three groups. The flowchart of participant enrollment is shown in Fig. 1. Table 1 summarizes the baseline clinical characteristics and procedural details of the study cohort.

\section{IVUS Findings}

IVUS was taken as the gold standard. Twenty IVUS pullbacks were from both the LAD and LCX to LMCA, 145 pullbacks were just from the LAD to LMCA. only 89 patients (54\%) were demonstrated to have an RI on IVUS (IVUS-RIs), 32 patients (19\%) were identified as having an h-D on IVUS (IVUS-h-Ds) and 44 patients (27\%) were considered to have an h-OM on IVUS (IVUS-hOMs) (Fig. 2a). The distance between the entrance of the IVUS-h-D and the carina of the furcation ranged from 0.5 to $2 \mathrm{~mm}$ (Fig. 2b). In some cases, the ostial IVUSh-D was very close to the carina of the furcation, which may explain why it was easily misjudged as RI on CAG.

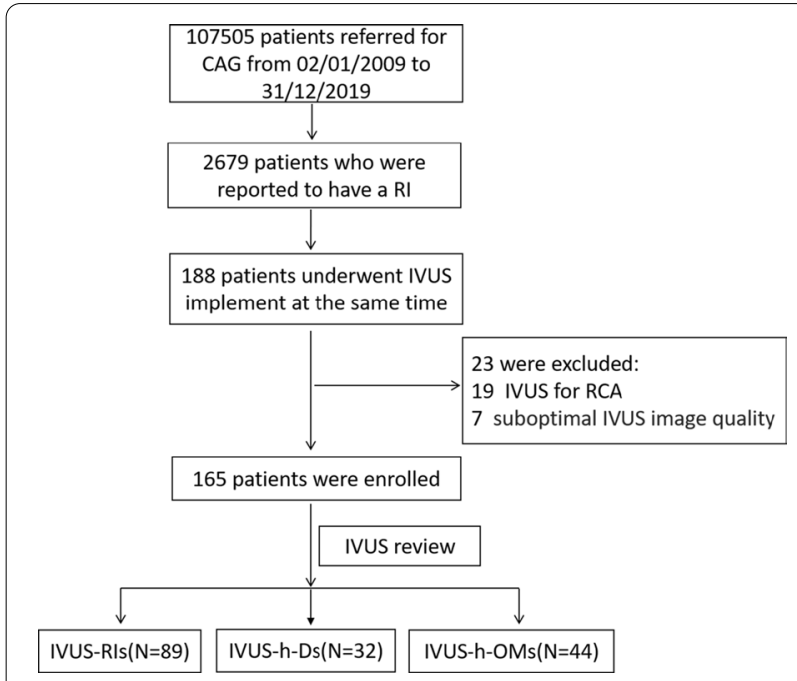

Fig. 1 Flowchart of participant enrollment and grouping. The study ultimately included 165 patients. These patients were grouped according to the results of their IVUS reviews, and in every group, only patients who underwent LMCA-LAD crossover stent deployment were included for comparison. Rl, ramus intermedius; $h-D$, high-origin diagonal branch; h-OM, high-origin obtuse marginal artery; LAD, left anterior descending branch; LMCA, left main coronary artery; IVUS, intravascular ultrasonography
Table 1 Baseline characteristics of 165 patients undergoing IVUS analysis

\begin{tabular}{ll}
\hline Variable & N (\%) \\
\hline Age, years & $64.8 \pm 10.4$ \\
Female (\%) & $26(16)$ \\
Current smoker (\%) & $79(48)$ \\
Disease history & \\
Hyperlipidemia (\%) & $14(8)$ \\
Hypertension (\%) & $64(39)$ \\
Diabetes Mellitus (\%) & $27(17)$ \\
PCI history (\%) & $46(28)$ \\
CABG history (\%) & $3(2)$ \\
Treatment & \\
Medicine alone (\%) & $25(15)$ \\
PCI (\%) & $140(75)$ \\
$\quad$ Trifurcation (\%) & $93(66)^{\mathrm{a}}$ \\
$\quad$ Crossover strategy (\%) & $84(90)^{\mathrm{b}}$ \\
\hline
\end{tabular}

$\mathrm{PCl}$, percutaneous coronary intervention; $\mathrm{CABG}$, coronary artery bypass graft

${ }^{\text {a }}$ Among 140 patients accepted $\mathrm{PCl}$ treatment, 93 patients had trifurcation lesion

b 84 of 93 trifurcation lesion were treated by LMCA-LAD crossover stenting

Figure 3 shows examples of IVUS-RI, IVUS-h-D and IVUS-h-OM in CAG (before and after PCI), and the diagrams of IVUS-RI, IVUS-h-D and IVUS-h-OM are shown in the bottom panel of Fig. 3. Figure 4 illustrates a series of IVUS cross-sections (1 mm apart) simulating a pullback from LAD to LMCA, for IVUS-RI, IVUS-h-D and IVUS-h-OM, respectively.

\section{The impact of RI on revascularization strategy}

In general, one-stent crossover stenting is considered the standard method for most coronary bifurcation lesions. In our study, 90\% (84/93) of LMCA furcation lesions were treated with LMCA-LAD crossover stenting. To evaluate the impact of crossover stenting on different angiographically judged RIs (CAG-RIs) and the impact of CAG-RI on the revascularization strategy for LM furcation, we divided the 84 patients who underwent LMCALAD one-stent crossover stenting into an IVUS-RI group $(\mathrm{N}=48)$, an IVUS-h-D group $(\mathrm{N}=12)$, and an IVUS-hOM group $(\mathrm{N}=24)$. The clinical characteristics and procedural details of these 84 patients are shown in Table 2.

There were 31 patients (65\%) in the IVUS-RI group (29 treated with the jailed wire technique, 2 with the jailed balloon technique), 6 patients (50\%) in the IVUS-h-D group ( 5 jailed wire and 1 jailed balloon procedure), and 12 patients $(50 \%)$ in the IVUS-h-OM group (all treated with the jailed wire technique) for whom an "RI" protective technique was used. Seven patients (14.6\%) in the IVUS-RI group and 4 patients (33.3\%) in the IVUS-hD group had CAG-RI compromise, while no patient in 
a

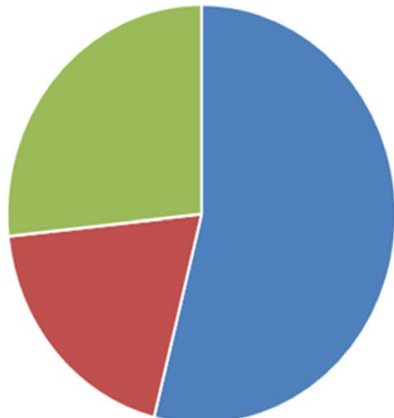

- CAG-h-D

II CAG-h-OM

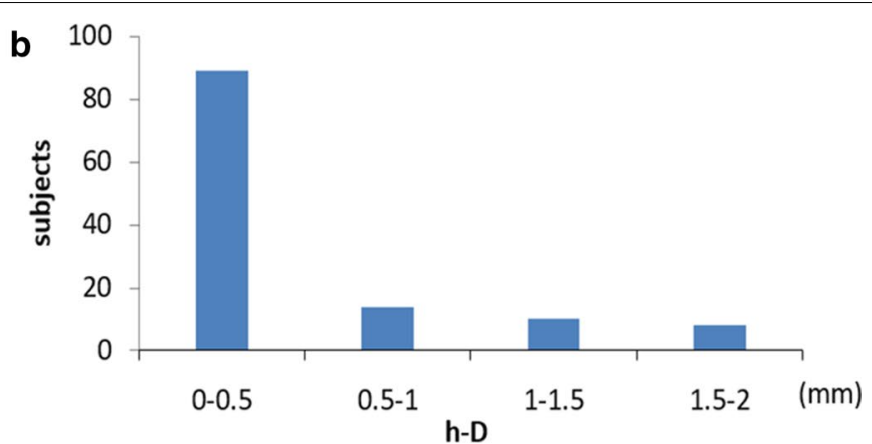

Fig. 2 Proportions of IVUS-RI, IVUS-h-D and IVUS-h-OM in IVUS views and the distribution characteristics of IVUS-h-D on IVUS. a The pie chart shows that only $54 \%$ of CAG-RIs were confirmed by IVUS, and the proportions of IVUS-h-D and IVUS-h-OM were $19 \%$ and $27 \%$, respectively. b Distance from ostial h-D to the LM furcation carina (mm). Rl, ramus intermedius; h-D, high-origin diagonal branch; h-OM, high-origin obtuse marginal artery; IVUS, intravascular ultrasonography; LM, left main

the IVUS-h-OM group had CAG-RI compromise; there was a significant difference among the three groups in this respect $(P=0.02)$. Five of 7 patients in the IVUS-RI group and 2 of 4 patients in the IVUS-h-D group received rescue balloon inflation. There was no significant difference between the two groups regarding the rate of jailed wire/balloon use or the rate of rescue balloon inflation ( $\mathrm{P}>0.05$ for both) (Table 2).

Seven of the 11 patients (63.6\%) with CAG-RI compromise, 42 of the 73 patients without CAG-RI compromise underwent the jailed wire/balloon procedure. There was no significant difference about the rate of jailed wire/ balloon technique between the two groups of patients. Table 3 summarized the possible factors of RI compromise in the patients with and without RI compromise. Stenosis of RI was higher in patients with RI compromise compared with those without RI compromise (60 [45$65]$ vs. $30[0-50] \%, P<0.01)$. As shown in Table 4 , there was a significant association between the stenosis of RI and RI compromise $(P=0.049)$. After additional correction for age and sex, it was still borderline significant $(P=0.051)$, which may be caused by the low sample size. Each 10\% increment in RI stenosis increased the risk of RI compromise by $25 \%$.

\section{Discussion}

The current study found that only $54 \%$ of angiographically judged RIs were confirmed by IVUS; IVUS-h-OM is rarely occluded during LMCA-LAD crossover stenting. Our findings suggest that preintervention IVUS is necessary to distinguish IVUS-h-OM from IVUS-RI and IVUS-h-D, and the revascularization strategy also needs to be tailored to different types of CAG-RIs.

Typically, LM bifurcates in LAD and LCX. Sometimes, an additional artery, known as RI, arises at the bifurcation of the LM, forming a trifurcation $[17,18]$. RI can be identified by CAG; however, standard angiographic projections of CAG are often associated with vessel foreshortening and anatomical overlap [12]. The rate of RI detection was lower on CAG than on CTA. In our study, only $2.5 \%$ of patients who underwent CAG were reported to have RIs, lower than the $20 \%$ observed by CTA $[3,4]$. The shadowgraphic nature of CAG are the varies diameter of RI may explain the low occurrence rate of RI reported by CAG. Furthermore, the interventional cardiologists often pay little attention to the non- target vessel or ignore RI in emergency ACS cases. These are also the reasons why the occurrence rate of RI reported by CAG is low. In contrast to the two-dimensional, shadowgraphic nature of coronary angiography, IVUS is an accurate tomographic technique for anatomical evaluation of the coronary artery [19]. It can accurately distinguish among RI, h-D, and h-OM, helping interventional cardiologists choose stenting and branch protection strategies. In this study of 165 LMCA-LAD IVUS images, we demonstrated that only $54 \%$ of CAG-RIs were confirmed as IVUS-RI, 32 CAG-RIs were identified as IVUS-h-D (19\%) and 44 CAG-RIs were identified as IVUS-h-OM (27\%).

The possible reasons for the lower detection rate of CAG are as follows: (1) RI has a similar course and perfusion region to h-OM or h-D, and it is easily misjudged. (2) Due to the different course of the coronary artery, the distal LM furcation and proximal LAD/LCX of many patients cannot be fully viewed in the conventional left anterior oblique caudal view or right anterior oblique caudal view, which leads to an unclear display of the ostial RI and can cause misjudgment [20]. (3) The conventional projection angle for LMCA furcation in our hospital is LAO $45^{\circ} / \mathrm{CAU} 30^{\circ}$, whereas Kocka et al. [21] reported that optimal fluoroscopic viewing angles for the LMCA bifurcation were LAO $0^{\circ} / \mathrm{CAU} 49^{\circ}$ in CTA imaging. This means that a significant proportion of bifurcation views 

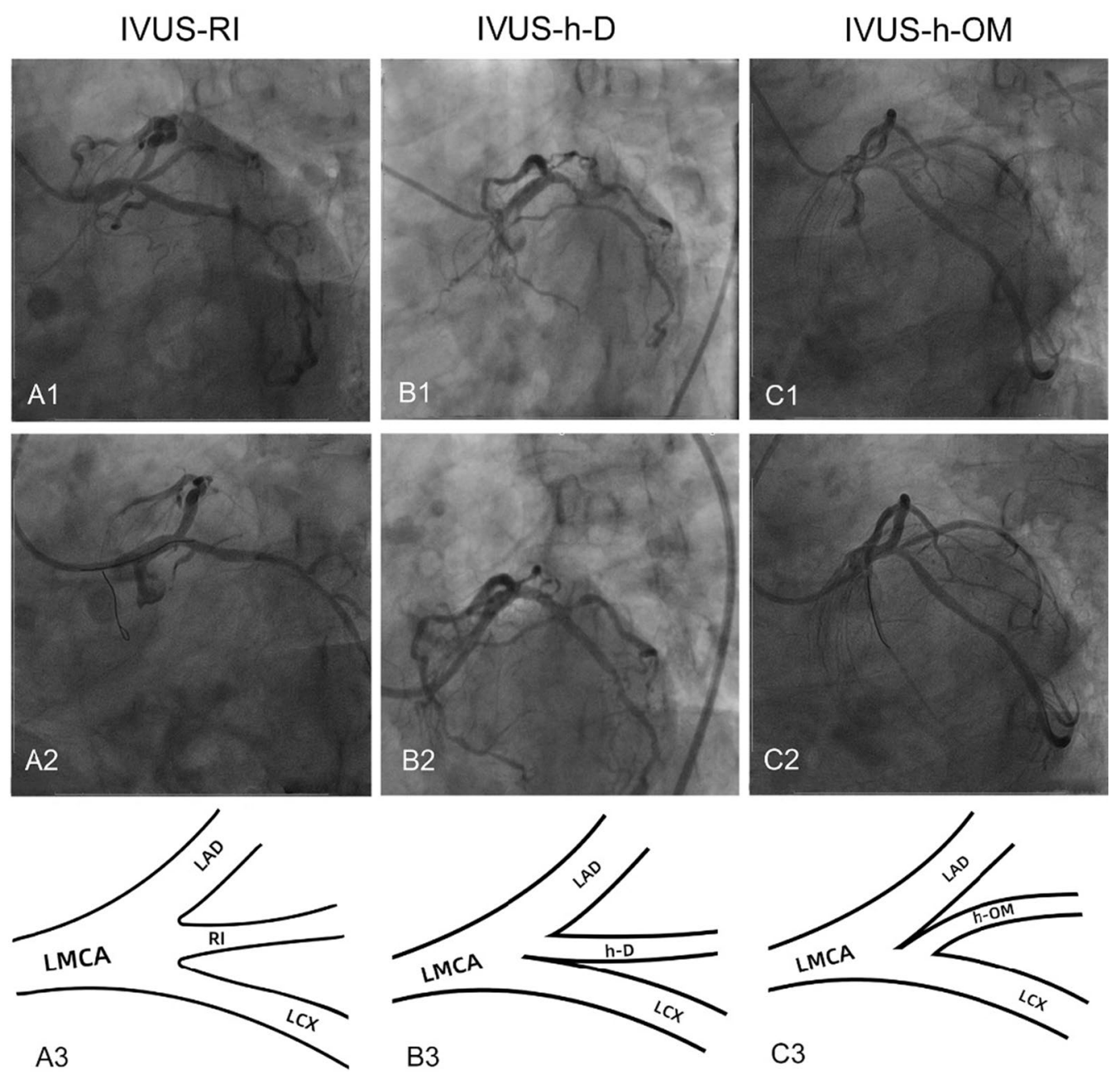

Fig. 3 Examples of IVUS-RI, IVUS-h-D and IVUS-h-OM in CAG and their diagrams. Top panel: A left anterior oblique caudal view ("spider" view) revealed a CAG-RI in the distal LM furcation in each group. Second panel: Different outcomes in groups treated with different revascularization strategies; A2: no jailed wire was used in IVUS-RI, and RI occlusion occurred; B2: a jailed wire was used in IVUS-h-D, and no slow flow or lumen reduction occurred; C2: no jailed wire was used in IVUS-h-OM, and no slow flow or lumen reduction occurred. Bottom panel: Diagrams of IVUS-RI, IVUS-h-D and IVUS-h-OM; A3: RI results from the trifurcation point; it can be seen at the entrance of the LCX, and the three lumens have blood flow; B3: IVUS-h-D was fully incorporated into the LAD before the LCX entered; C3: IVUS-h-OM was fully incorporated into the LCX before the LCX entered, but it cannot be seen from the LMCA-LAD IVUS view. Rl, ramus intermedius; $h-D$, high-origin diagonal branch; h-OM, high-origin obtuse marginal artery; LAD, left anterior descending branch; LCX, left circumflex artery; CAG, coronary artery angiography; IVUS, intravascular ultrasonography; LMCA, left main coronary artery

lie outside the practical projection range. (4) Interventional cardiologists do not pay attention to RI, especially when the target lesion is not at the LMCA furcation. (5) Even if an interventional cardiologist wishes to change the fluoroscopic viewing angles to observe LM furcation clearly, not all CTA-defined fluoroscopic viewing angles are practical or achievable with existing $\mathrm{C}$-arm equipment across patients.

PCI procedures for LM bifurcation lesions are still difficult because the jailed wire or jailed balloon technique must be used [9, 10, 22]. In general, one-stent crossover stenting is considered the standard method for most coronary bifurcation lesions [23, 24]. However, it is associated with a risk of SB occlusion after MV stenting [25] because of a combination of carina shift and plaque shift into the SB [26, 27]. Intervention for a trifurcation lesion is more complicated, requiring more wires and various complex interventional techniques [28]. Studies have shown an increase in periprocedural complications (dissection, acute side branch occlusion, periprocedural myocardial infarction) in trifurcation diseases [29, 30]. Among the 84 patients who underwent LMCA-LAD 


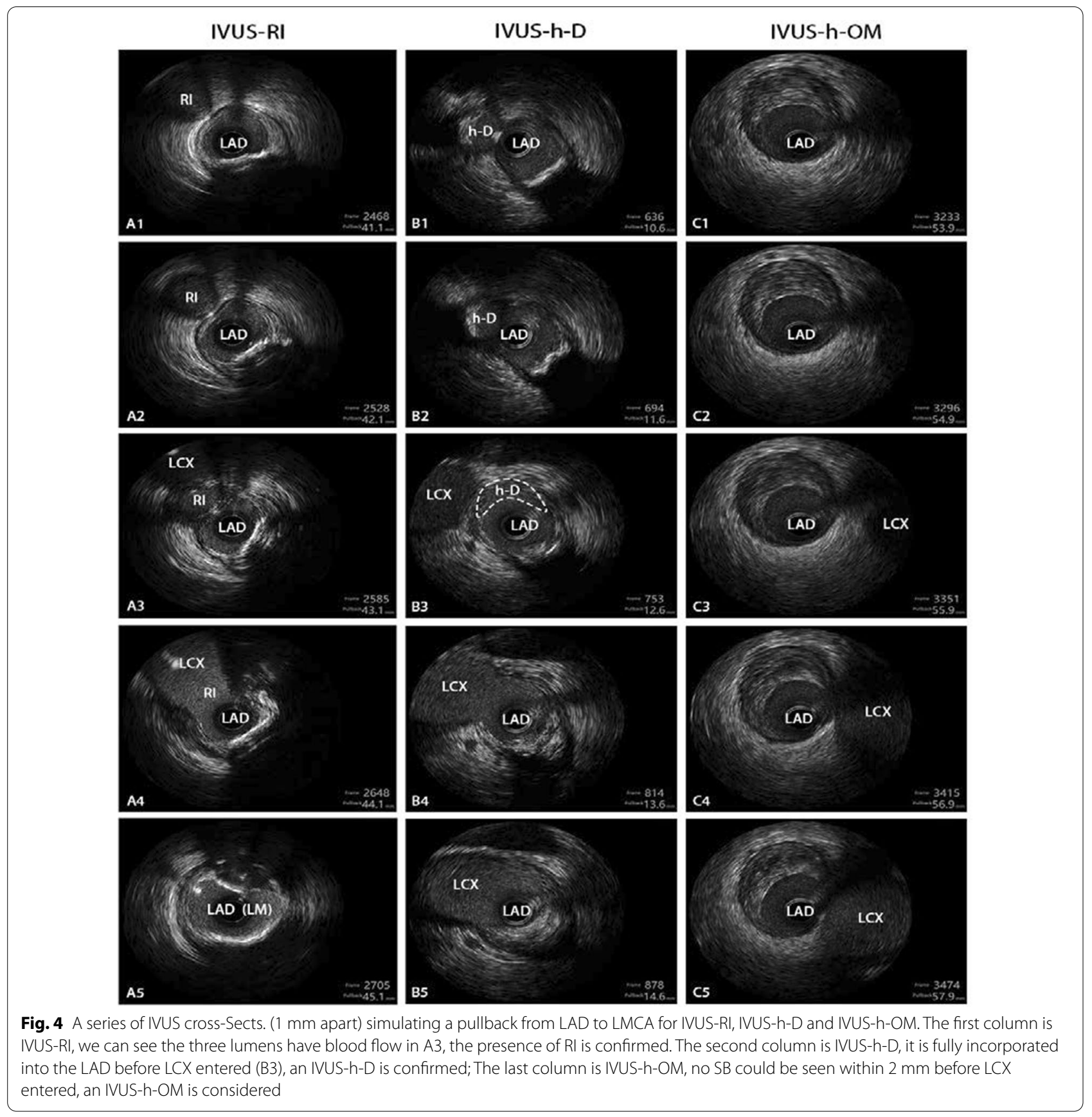

one-stent crossover stenting, 7 patients $(14.6 \%)$ in the IVUS-RI group, 4 patients (33.3\%) in the IVUS-h-D group and no patients $(0 \%)$ in the IVUS-h-OM group had CAG-RI compromise (narrowing/occlusion) $(P=0.02)$. The probability of compromise is more than twice as high in IVUS-h-D patients as in IVUS-RI patients. However, if the CAG-RI is actual an IVUS-h-OM, the probability of compromise will be lower after LMCA-LAD crossover stenting.
There may be a lot of cofounding factors that influence RI compromise. To discuss the risk of RI compromise, univariate and multivariate analysis were performed. There were no significant differences about the rate of jailed wire/balloon technique, plaque burden of LAD, post-ballooning pressure between patients with and without CAG-RI compromise. However, the stenosis of CAG$\mathrm{RI}$ in patients with RI compromise was higher compared with those without RI compromise. Multivariate analysis 
Table 2 Baseline and operation Characteristics of patients who accepted LMCA-LAD crossover stenting in the three groups

\begin{tabular}{|c|c|c|c|c|c|}
\hline & $\begin{array}{l}\text { Total } \\
(\mathrm{N}=84)\end{array}$ & $\begin{array}{l}\text { IVUS-RIs group } \\
(\mathrm{N}=48)\end{array}$ & $\begin{array}{l}\text { IVUS-h-Ds group } \\
(\mathrm{N}=12)\end{array}$ & $\begin{array}{l}\text { IVUS-h-OMs group } \\
(\mathrm{N}=24)\end{array}$ & $P$ value \\
\hline Age, years & $64.1 \pm 11.0$ & $64.0 \pm 10.9$ & $72.4 \pm 8.6$ & $60.2 \pm 10.4$ & 0.32 \\
\hline Female (\%) & $7(8.3)$ & $3(6.3)$ & $0(0)$ & $4(16.7)$ & 0.17 \\
\hline Current smoker (\%) & $40(47.6)$ & $22(45.8)$ & $5(41.7)$ & $13(54.1)$ & 0.72 \\
\hline \multicolumn{6}{|l|}{ Diseases history } \\
\hline Hypertension (\%) & $33(39.3)$ & 19 (39.6) & $4(33.3)$ & $10(41.7)$ & 0.89 \\
\hline Hyperlipidemia (\%) & $8(9.5)$ & $5(10.4)$ & $1(8.3)$ & $2(8.3)$ & 0.95 \\
\hline Diabetes mellitus (\%) & $14(16.7)$ & $7(14.5)$ & $2(16.7)$ & $5(20.8)$ & 0.8 \\
\hline PCI history (\%) & $25(29.8)$ & $14(29.2)$ & $4(33.3)$ & $7(29.2)$ & 0.96 \\
\hline CABG history (\%) & $2(2.4)$ & $1(2.1)$ & $0(0)$ & $1(4.2)$ & 0.73 \\
\hline Medina (\%) & & & & & 0.57 \\
\hline $0,1,0$ & $46(54.8)$ & $26(54.2)$ & $6(50.0)$ & $14(58.3)$ & \\
\hline $0,1,1$ & $13(15.5)$ & $10(20.8)$ & $2(16.7)$ & $1(4.2)$ & \\
\hline $1,0,0$ & $6(7.1)$ & $2(4.2)$ & $1(8.3)$ & $2(8.3)$ & \\
\hline $1,1,0$ & $15(17.9)$ & $6(12.5)$ & $3(25.0)$ & $6(25.0)$ & \\
\hline $1,1,1$ & $5(6.0)$ & $4(8.3)$ & $0(0)$ & $1(4.2)$ & \\
\hline $\mathrm{Rl}, \%$ & $30(0-60)$ & $30(0-60)$ & $50(22.5-80)$ & $30(0-50)$ & 0.33 \\
\hline Plaque burden, $\%$ & $84.5(76-90)$ & $88(78.75-90.5)$ & $83.5(74-87.25)$ & $78.5(76-89)$ & 0.05 \\
\hline \multicolumn{6}{|l|}{$\mathrm{PCl}$} \\
\hline Stent size, $\mathrm{mm}$ & $3.5(3.5-3.5)$ & $3.5(3.0-3.5)$ & $3.5(3.5-3.5)$ & $3.5(3.5-3.625)$ & 0.83 \\
\hline Stent balloon pressure, atm & $12.0(12.0-14.0)$ & $12.0(12.0-14.0)$ & $12.0(12.0-12.0)$ & $12.0(12.0-12.0)$ & 0.24 \\
\hline Post balloon size, mm & $3.5(3.5-4)$ & $3.5(3.5-4.0)$ & $3.63(3.5-4)$ & $3.75(3.5-4)$ & 0.63 \\
\hline Post balloon pressure, atm & $18.0(16.0-20.0)$ & $18.0(15.0-20.0)$ & $20.0(19.5-20.0)$ & $18.0(16.0-18.5)$ & 0.12 \\
\hline CAG-RI protection & & & & & 0.40 \\
\hline Jailed wire, \% & $46(54.8)$ & $29(60.4)$ & $5(41.7)$ & $12(50.0)$ & \\
\hline Jailed balloon, \% & $3(4.8)$ & $2(2.1)$ & $1(8.3)$ & 0 & \\
\hline Radiation medium, ml & $210(167.5-300)$ & $220(180-301.25)$ & $190(157.5-220)$ & $200(167.5-302.5)$ & 0.27 \\
\hline Radiation, mGy & $900(780-1502.25)$ & $1005(795-1575)$ & $795(630-1062.5)$ & 835 (772.5-1531.75) & 0.22 \\
\hline Operation time, min & $100(80-120)$ & $110(87.5-130)$ & $85(80-112.5)$ & $90(80-120)$ & 0.24 \\
\hline \multicolumn{6}{|l|}{ Outcome } \\
\hline Combined outcome*, \% & $11(13.1)$ & $7(14.6)$ & $4(33.3)$ & $0(0)$ & 0.02 \\
\hline Rescue balloon inflated, \% & $7(8.3)$ & $5(10.4)$ & $2(8.3)$ & $0(0)$ & 0.17 \\
\hline
\end{tabular}

Continuous variables were shown as mean $\pm \mathrm{SD}$ or median (IQR) according to data distribution, and categorical variables were shown as $\mathrm{N}$ (\%). The $P$ values for intergroup differences were calculated using Kruskal-Wallis test for continuous variables and Chi-squared Test or Fisher exact test for categorical variables

*Severe stenosis of the RI ostium (>75\%) or significant RI flow impairment $(\mathrm{TIMI}<3$ ) or both

showed that the stenosis of RI was an independent risk factor of RI compromise $(P=0.049)$. Each $10 \%$ increment in RI stenosis increases the risk of RI compromise by $25 \%$. Therefore, not only the image characteristics of CAG-RI in IVUS, but also the stenosis severity of CAGRI should be paid attention to.

The jailed wire/balloon technique has been shown to improve the rates of $\mathrm{SB}$ reopening in the event of closure [31]. If too many guidewires are used, they will become entangled and make the PCI procedure more difficult. Hence, it is of great value to distinguish IVUS-RI and IVUS-h-D from IVUS-h-OM in LM trifurcation lesions. When crossover stenting is performed in LMCA-LAD for LM furcation lesions with IVUS-RI or IVUS-h-D, a jailed guidewire is needed to protect the IVUS-RI or IVUS-h-D, if the branch is large $(>2 \mathrm{~mm})$, or there is severe stenosis of IVUS-RI or IVUS-h-D, a jailed balloon may be used. For LM furcation lesions with IVUS-h-OM, except for LCX jailed wire procedures, no jailed wire is needed to protect the IVUS-h-OM, which will simplify the trifurcation lesion to a bifurcation lesion and thus reduce the volume of contrast agent, the amount of radiation exposure, and the procedural time. Therefore, in agreement with a recent meta-analysis [32], we suggest that preintervention IVUS should be performed 
Table 3 Characteristics of patients with and without RI compromise

\begin{tabular}{lll}
\hline & $\begin{array}{l}\text { Rl compromise } \\
(\mathbf{N}=\mathbf{1 1})\end{array}$ & $\begin{array}{l}\text { No RI compromises } \\
(\mathbf{N}=\mathbf{7 3})\end{array}$ \\
\hline Age, years & $67.6 \pm 11.5$ & $63.6 \pm 10.9$ \\
Female, \% & $0(0)$ & $7(9.6)$ \\
Stenosis of LAD, \% & $85.5 \pm 13.5$ & $88.7 \pm 11.1$ \\
Stenosis of RI, \% & $60(45-65)$ & $30(0-50)^{*}$ \\
Rl jailed wire/balloon, \% & $7(63.6)$ & $42(57.5)$ \\
Plaque burden of LAD, \% & $79.6 \pm 12.6$ & $83.1 \pm 9.6$ \\
Stent diameter, mm & $3.45 \pm 0.35$ & $3.47 \pm 0.37$ \\
Stent pressure, atm & $11.64 \pm 1.5$ & $12.36 \pm 1.6$ \\
Post balloon diameter, mm & $3.59 \pm 0.53$ & $3.72 \pm 0.5$ \\
Post balloon pressure, atm & $17.09 \pm 4.8$ & $17.86 \pm 3.33$ \\
\hline
\end{tabular}

Continuous variables were shown as mean $\pm \mathrm{SD}$ or median (IQR) according to data distribution, and categorical variables were shown as $\mathrm{N}(\%)$. Asterisk represented a $P$ value smaller than 0.05 for inter-group difference in Wilcox rank-sum test

Table 4 Multivariate analysis of risk factors for RI compromise

\begin{tabular}{llll}
\hline & Odd Ratio & $\mathbf{9 5 \% ~ C l}$ & P value \\
\hline Age, years & 1.05 & $0.98-1.12$ & 0.20 \\
Female, \% & 1.00 & $0.99-1.01$ & 0.99 \\
Stenosis of RI, \% & 1.26 & $1.01-1.61$ & $0.05^{*}$ \\
Rl jailed wire/balloon, \% & 1.97 & $0.53-7.50$ & 0.31 \\
Post-balloon pressure, atm & 0.91 & $0.74-1.12$ & 0.38 \\
Plaque burden of LAD, \% & 1.02 & $0.62-1.68$ & 0.92 \\
\hline
\end{tabular}

${ }^{*} P=0.049$. After additional correction for age and sex,$P=0.051$

in LM furcation lesions to distinguish among IVUS-RI, IVUS-h-D, and IVUS-h-OM, and the revascularization strategy also needs to be tailored to different types of CAG-RIs.

\section{Study limitations}

The study has inherent limitations owing to its singlecenter, retrospective design and relatively small sample size, which might introduce selection bias. In addition, the low occurrence rate of RI reported by CAG may be another selection bias. Third, IVUS was performed in LAD alone in most cases, and we could not evaluate the ostial LAD, RI and LCX simultaneously. Last, the plaque load of CAG-RI can also affect the RI blood flow after a one-stent crossover strategy stent implantation [33]. We did not evaluate the plaque burden in RI because of the retrospective design and the IVUS pullbacks were withdrawn from LAD to LMCA. Further prospective investigation is warranted to evaluate RI characteristics and its impact on PCI more accurately.

\section{Conclusions}

In conclusion, the current study illustrated that only $54 \%$ of CAG-RIs were confirmed by IVUS, which necessitates preintervention IVUS implementation in LM furcation lesions.

\section{Abbreviations \\ CAG: Coronary artery angiography; CTA: Computed tomography angiography; CRA: Cranial; CAU: Caudal; h-D: High-origin diagonal branch; h-OM: High- origin obtuse marginal artery; IVUS: Intravascular ultrasonography; LAD: Left anterior descending artery; LAO: Left anterior oblique; LCX: Left circumflex artery; LMCA: Left main coronary artery; MV: Main vessel; PCl: Percutaneous coronary intervention; RAO: Right anterior oblique; RI: Ramus intermedius; SB: Side branch. \\ Acknowledgements \\ The authors would like to thank the research staff for their help.}

\section{Authors' contributions}

XG and ZH drafted the manuscript and reviewed the IVUS images. ZS did statistical analysis. QW and JQ helped revising the manuscript for important intellectual content. LG and JG were involved in supervisory role in study design. All authors have read, revised and approved the final manuscript.

Funding

This work was supported by the National Natural Science Foundation of China (82070281, 81870269).

\section{Availability of data and materials}

All data and material used and/or analyzed during the current study are available from the corresponding author on reasonable request.

\section{Declarations}

Ethics approval and consent to participate

No treatment was tested in patients by the authors for this article. The Ethics Committee of Zhongshan Hospital affiliated with Fudan University had approved the work and received all the written informed consent. All methods were performed in accordance with the relevant guidelines and regulations.

\section{Consent for publication}

Not applicable.

\section{Competing interests}

All authors declare that they have no competing financial interests.

\section{Author details}

'Department of Cardiology, Deltahealth Hospital, Shanghai 201702, People's Republic of China. ${ }^{2}$ Department of Cardiology, Shanghai Institute of Cardiovascular Disease, Zhongshan Hospital, Fudan University, 180 Fenglin Road, Shanghai 200032, People's Republic of China. ${ }^{3}$ Human Phenome Institute, Fudan University, Shanghai 200438, People's Republic of China.

Received: 20 February 2021 Accepted: 16 April 2021

Published online: 30 April 2021

\section{References}

1. Kini S, Bis KG, Weaver L. Normal and variant coronary arterial and venous anatomy on high-resolution CT angiography. AJR Am J Roentgenol. 2007;188(6):1665-74.

2. Wrobel G, Spalek M, Spalek J, Kuder T. Dual left anterior descending coronary artery (type III) and the presence of myocardial bridges-a post-mortem examination. Folia Morphol (Warsz). 2020;79(3):634-9. 
3. O'Brien JP, Srichai MB, Hecht EM, Kim DC, Jacobs JE. Anatomy of the heart at multidetector CT: what the radiologist needs to know. Radiographics. 2007;27(6):1569-82.

4. Kosar P, Ergun E, Ozturk C, Kosar U. Anatomic variations and anomalies of the coronary arteries: 64-slice CT angiographic appearance. Diagn Interv Radiol. 2009;15(4):275-83.

5. Mathai RT, Fahmy DM, Sadek HL, Renno WM. Congenital coronary artery anomalies in adult population detected using dual source ECG-gated CTA in a single institution. Folia Morphol (Warsz). 2017;76(2):208-18

6. Testa L, Bedogni F. How should I treat a coronary "traffic jam"? A triple kissing balloon in distal left main trifurcation. Eurolntervention. 2011;6(8):1011-6.

7. Park DW, Ahn JM, Park SJ, Taggart DP. Percutaneous coronary intervention in left main disease: SYNTAX, PRECOMBAT, EXCEL and NOBLE-combined cardiology and cardiac surgery perspective. Ann Cardiothorac Surg. 2018;7(4):521-6.

8. Gershlick AH, Kandzari DE, Banning A, Taggart DP, Morice MC, Lembo NJ, Brown WM 3rd, Banning AP, Merkely B, Horkay F, et al. Outcomes after left main percutaneous coronary intervention versus coronary artery bypass grafting according to lesion site: results from the EXCEL trial. JACC Cardiovasc Interv. 2018;1 1(13):1224-33.

9. Collet C, Mizukami T, Grundeken MJ. Contemporary techniques in percutaneous coronary intervention for bifurcation lesions. Expert Rev Cardiovasc Ther. 2018;16(10):725-34.

10. Milasinovic D, Wijns W, Ntsekhe M, Hellig F, Mohamed A, Stankovic G. Step-by-step manual for planning and performing bifurcation PCI: a resource-tailored approach. Eurolntervention. 2018;13(15):e1804-11.

11. Furuichi S, Sangiorgi GM, Palloshi A, Godino C, Airoldi F, Montorfano M, Chieffo A, Michev I, Carlino M, Colombo A. Drug-eluting stent implantation in coronary trifurcation lesions. J Invasive Cardiol. 2007;19(4):157-62.

12. Zheng $\mathrm{S}$, Meiying T, Jian S. Sequential reconstruction of vessel skeletons from X-ray coronary angiographic sequences. Comput Med Imaging Graph. 2010;34(5):333-45.

13. Rubinshtein R, Lerman A, Spoon DB, Rihal CS. Anatomic features of the left main coronary artery and factors associated with its bifurcation angle: a 3-dimensional quantitative coronary angiographic study. Catheter Cardiovasc Interv. 2012;80(2):304-9.

14. De la Torre Hernandez JM, Garcia Camarero T. Intravascular ultrasound for the diagnosis and treatment of left main coronary artery disease. Interv Cardiol Clin. 2015;4(3):361-81.

15. Neumann FJ, Sousa-Uva M, Ahlsson A, Alfonso F, Banning AP, Benedetto U, Byrne RA, Collet JP, Falk V, Head SJ, et al. 2018 ESC/EACTS guidelines on myocardial revascularization. Kardiol Pol. 2018;76(12):1585-664.

16. Hildick-Smith D, Lassen JF, Albiero R, Lefevre T, Darremont O, Pan M, Ferenc M, Stankovic G, Louvard Y, European Bifurcation C. Consensus from the 5th European Bifurcation Club meeting. Eurolntervention. 2010;6(1):34-8.

17. Angelini P. Coronary artery anomalies: an entity in search of an identity. Circulation. 2007;115(10):1296-305.

18. Pereira da Costa Sobrinho O, Dantas de Lucena J, Silva Pessoa R, Andrade Verissimo N, Martins Nunes L, Karline Rojas P, Simas Macedo E, Erivan Facanha Barreto J, Lopes Ribeiro Junior H, Viana Gondim D, et al. Anatomical study of length and branching pattern of main trunk of the left coronary artery. Morphologie. 2019;103(341):17-23.

19. Mintz GS, Lefevre T, Lassen JF, Testa L, Pan M, Singh J, Stankovic G, Banning AP. Intravascular ultrasound in the evaluation and treatment of left main coronary artery disease: a consensus statement from the European Bifurcation Club. Eurolntervention. 2018;14(4):e467-74.

20. Kočka V, Thériault-Lauzier P, Xiong T-Y, Ben-Shoshan J, Petr R, Laboš M, Buithieu J, Mousavi N, Pilgrim T, Praz F, et al. Optimal fluoroscopic projections of coronary ostia and bifurcations defined by computed tomographic coronary angiography. JACC Cardiovasc Interv. 2020;13(21):2560-70

21. Kocka V, Theriault-Lauzier P, Xiong TY, Ben-Shoshan J, Petr R, Labos M, Buithieu J, Mousavi N, Pilgrim T, Praz F, et al. Optimal fluoroscopic projections of coronary ostia and bifurcations defined by computed tomographic coronary angiography. JACC Cardiovasc Interv. 2020;13(21):2560-70.

22. Lefevre T, Girasis C, Lassen JF. Differences between the left main and other bifurcations. Eurolntervention. 2015;11 Suppl V:V106-110.

23. Hildick-Smith D, de Belder AJ, Cooter N, Curzen NP, Clayton TC, Oldroyd KG, Bennett L, Holmberg S, Cotton JM, Glennon PE, et al. Randomized trial of simple versus complex drug-eluting stenting for bifurcation lesions: the British Bifurcation Coronary Study: old, new, and evolving strategies. Circulation. 2010;121(10):1235-43.

24. Banning AP, Lassen JF, Burzotta F, Lefevre T, Darremont O, Hildick-Smith $D$, Louvard Y, Stankovic G. Percutaneous coronary intervention for obstructive bifurcation lesions: the 14th consensus document from the European Bifurcation Club. Eurolntervention. 2019;15(1):90-8.

25. Sakamoto N, Hoshino Y, Mizukami H, Sugimoto K, Yamaki T, Kunii H, Nakazato K, Suzuki H, Saitoh S, Takeishi Y. Intravascular ultrasound predictors of acute side branch occlusion in coronary artery bifurcation lesions just after single stent crossover. Catheter Cardiovasc Interv. 2016;87(2):243-50.

26. Koo BK, Waseda K, Kang HJ, Kim HS, Nam CW, Hur SH, Kim JS, Choi D, Jang Y, Hahn JY, et al. Anatomic and functional evaluation of bifurcation lesions undergoing percutaneous coronary intervention. Circ Cardiovasc Interv. 2010;3(2):113-9.

27. Xiu J, Choi SY, Mintz GS, Araki H, Masuda N, Morino Y, Sonoda S, Tahk SJ, Ochiai M, Maehara A. Three-dimensional intravascular ultrasound evaluation of carina and plaque shift at the distal left main coronary artery bifurcation after treatment with a one-stent cross-over technique. Catheter Cardiovasc Interv. 2013:81(7):1142-9.

28. Hussein $\mathrm{H}$, Fang HY, Wu CJ. Transradial complex left main trifurcation intervention. Catheter Cardiovasc Interv. 2010;76(5):679-83.

29. Lopez-Pena U, Garcia-Garcia F, Robledo-Nolasco R, Fernandez-Cesena E, Borrego-Montoya R, Morales N. Distal left main trifurcation disease in a patient with porcelain aorta. Cardiovasc Revasc Med. 2017;18(5S1):S27-9.

30. Grundeken MJ, Wykrzykowska JJ, Ishibashi Y, Garg S, de Vries T, GarciaGarcia HM, Onuma Y, de Winter RJ, Buszman P, Linke A, et al. First generation versus second generation drug-eluting stents for the treatment of bifurcations: 5-year follow-up of the LEADERS all-comers randomized trial. Catheter Cardiovasc Interv. 2016;87(7):E248-260.

31. Hahn JY, Chun WJ, Kim JH, Song YB, Oh JH, Koo BK, Rha SW, Yu CW, Park JS, Jeong JO, et al. Predictors and outcomes of side branch occlusion after main vessel stenting in coronary bifurcation lesions: results from the COBIS II Registry (COronary Blfurcation Stenting). J Am Coll Cardiol. 2013:62(18):1654-9.

32. de la Torre Hernandez JM, Baz Alonso JA, Gomez Hospital JA, Alfonso Manterola F, Garcia Camarero T, Gimeno de Carlos F, Roura Ferrer G, Recalde AS, Martinez Luengas IL, Gomez Lara J, et al. Clinical impact of intravascular ultrasound guidance in drug-eluting stent implantation for unprotected left main coronary disease: pooled analysis at the patientlevel of 4 registries. JACC Cardiovasc Interv. 2014;7(3):244-54.

33. Kang SJ, Mintz GS, Kim WJ, Lee JY, Park DW, Lee SW, Kim YH, Lee CW, Park SW, Park SJ. Preintervention angiographic and intravascular ultrasound predictors for side branch compromise after a single-stent crossover technique. Am J Cardiol. 2011;107(12):1787-93.

\section{Publisher's Note}

Springer Nature remains neutral with regard to jurisdictional claims in published maps and institutional affiliations. 